\title{
Response of Crown Ether Functionalized Polythiophenes to Alkaline lons
}

\author{
David Zanuy, $^{\dagger}$ Julien Preat, $^{\dagger, \S}$ Eric A. Perpète, ${ }^{\perp, \|}$ and Carlos Alemán ${ }^{*}, \dagger, \dagger$ \\ ${ }^{\dagger}$ Departament d’Enginyeria Química, E. T. S. d’Enginyeria Industrial de Barcelona, Universitat Politècnica de Catalunya, Diagonal \\ 647, 08028 Barcelona, Spain \\ ${ }^{\ddagger}$ Center for Research in Nano-Engineering, Universitat Politècnica de Catalunya, Campus Sud, Edifici C’, C/Pasqual i Vila s/n, \\ Barcelona E-08028, Spain \\ ${ }^{\perp}$ Unité de Chimie Physique Théorique et Structurale (2742), Facultés Universitaires Notre-Dame de la Paix, rue de Bruxelles, 61, \\ B-5000 Namur, Belgium
}

ABSTRACT: The sensing response of 15-crown-5-ether functionalized polythiophene to $\mathrm{Li}^{+}, \mathrm{Na}^{+}$, and $\mathrm{K}^{+}$has been investigated at the atomistic level using molecular dynamics simulations. The stability associated with all the identified binding sites has been corroborated by quantum mechanical calculations. Although the cavity of the macrocycle is not the most visited binding site, such receptor is responsible of the selective sensing response of this polythiophene derivative. $\mathrm{PF}_{6}{ }^{-}$counterions reduce the mobility of the alkaline cations, which do not occupy the crown ether cavity of consecutive repeating units due to electrostatic repulsions. Furthermore, the relative entropy for the "free state $\rightarrow$ bound state" has been estimated using a procedure based on the covariance matrix atom-positional fluctuations. Evaluation of the entropic contributions allow us to complete the thermodynamics scenario of binding process, which was recently initiated by calculating the enthalpies at quantum mechanical level [Chem. Eur. J. 2009, 15, 4676]. Results indicate an entropycally driven binding

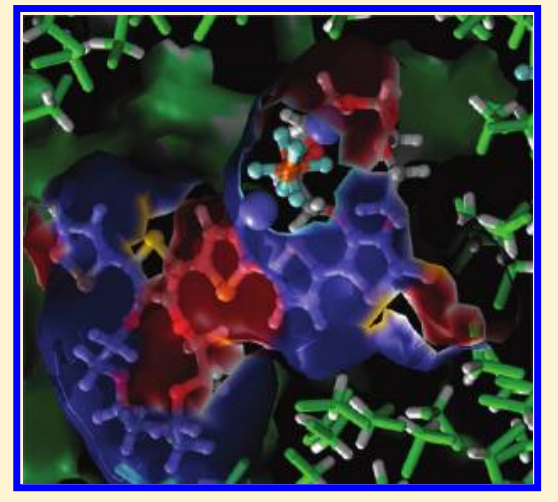
preference.

\section{INTRODUCTION}

Selective host-guest interactions in combination with conducting polymers are exploited in iono-electronic devices, in which the complexation $\leftrightarrow$ decomplexation processes allow an externally induced switching between two different states. ${ }^{1-3}$ This phenomenon produces a detectable response, which is based on the transduction of chemical information (i.e., the formation of specific interactions) into the change of an electrical signal (i.e. the change of the electronic properties of the conjugated backbone). Crown ether-functionalized materials are particularly interesting for these applications due to their strong ion-dipole interactions with metal cations. In this respect different polythiophenes (PThs) with crown ether macrocycles directly fused to the heterocycles or linked to them through suitable spacers have been reported. Specifically, after Sone and co-workers prepared in 1989 the first crown ether functionalized thiophenes, ${ }^{4}$ several modifications to improve their sensory performance were developed without success. ${ }^{5-7}$ However, crown ethers functionalized PThs displaying a satisfactory sensory response toward alkaline cations were reported by the groups of Bäuerle, ${ }^{8-12}$ Swager, $^{13-15}$ and Roncali. ${ }^{16-19}$

In spite of the interesting properties of crown ether functionalized PThs, the microscopic information about both the sensing mechanism and the response of the polymer chains toward the presence of alkaline cations in the cavities of the macrocycles is very scarce. Within this context, we recently investigated the strength of the binding between alkaline ions
$\left(\mathrm{Li}^{+}, \mathrm{Na}^{+}\right.$, and $\left.\mathrm{K}^{+}\right)$and a functionalized PTh bearing a 15crown-5-ether macrocycle (PTn-15c5e; Scheme 1), ${ }^{20}$ which

Scheme 1. Chemical Structure of the PTn-15c5e Sensor

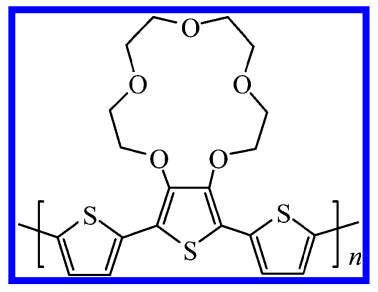

was found to be particularly successful in ion sensing. ${ }^{9,10}$ For this purpose, quantum mechanical (QM) calculations and molecular dynamics (MD) simulations in dichloromethane were performed using model complexes involving one repeating unit (i.e., $n=1$ in Scheme 1). QM calculations evidenced that the attractive character between neutral PTn$15 \mathrm{c} 5 \mathrm{e}$ and alkaline ions increases as the size of the ion decreases. Moreover, MD simulations allowed us to explain the experimental selectivity of $\mathrm{Na}^{+}$with respect to $\mathrm{Li}^{+}$, which was attributed to the entropic contribution.

Received: October 17, 2011

Revised: March 21, 2012

Published: April 5, 2012 
However, important microscopic information related with the response of crown ether-functionalized PThs against alkaline ions remains unknown yet. In this work, we perform classical MD simulations at the atomistic level of PT $n-15 \mathrm{c} 5 \mathrm{e}(n$ $=1$ and 6) complexed with $\mathrm{Li}^{+}, \mathrm{Na}^{+}$and $\mathrm{K}^{+}$in dichloromethane-solution to investigate different unknown aspects of the sensing response of this PTh derivative to alkaline ions. The Results and Discussion is organized in five different subsections. In the first one, we analyze the binding sites of the different alkaline ions. Our results indicate that the more visited binding sites are the external side of macrocycle and above/below the nonfunctionalized thiophene rings rather than the cavity of the receptor, as is typically assumed. In spite of this, the second subsection, in which the dynamics of the different binding sites is analyzed, shows that the selectivity of crown ether functionalized PTh is defined by the confinement of the cation within the cavity of the macrocycle. Next, we show that solvent molecules surrounding alkaline ions are rapidly exchanged with those of the bulk. After this, the fourth subsection presents the evaluation of relative entropy for the binding process using the information recorded from the different $\mathrm{MD}$ simulations. Finally, we discuss and compare results derived from quantum mechanical calculations on PT1-15c5e $\cdots \mathrm{M}^{+}$complexes $\left(\mathrm{M}^{+}=\right.$ $\mathrm{Li}^{+}, \mathrm{Na}^{+}$, and $\mathrm{K}^{+}$), in which the alkaline ion is accommodated in the cavity or in the external side of the macrocycle.

\section{METHODS}

Molecular Systems. For each alkaline ion MD simulations were performed using an all-atom approximation and considering two molecular systems, which differ in the number of repeating units used to describe PTn-15c5e. The first system involved a sensor with 18 thiophene rings ( $n=6$ in Scheme 1; PT6-15c5e) while the second one was constituted by 3 thiophene rings $(n=1$; PT1-15c5e). In each simulation the PT $n$-15c5e molecule was placed in the center of an orthorhombic simulation box $(a=120.0 \AA, b=c=100.0 \AA$ and $a=b=c=42.0 \AA$ for $n=6$ and 1, respectively) filled with solvent molecules, which was previously equilibrated in NPT conditions (temperature $298 \mathrm{~K}$ and 1 atm of pressure). Once inside the box, solvent molecules overlapped with any atom of the PT $n-15 \mathrm{c} 5 \mathrm{e}$ chain were removed, the number of solvent molecules being 9518 and 577 for systems with $n=6$ and 1, respectively. After this, an alkaline ion was introduced in the cavity of each PTn-15c5e receptor (i.e., six and one for $n=6$ and 1 , respectively). The electric neutrality was reached by adding the counterions (6 and 1 anions for PT6-15c5e and PT1-15c5e, respectively) to the simulation box. Initially, $\mathrm{PF}_{6}{ }^{-}$ counterions were put in positions relatively close to the alkaline ions (i.e., at a distance lower than $4 \AA$ ).

Two independent simulations in NPT conditions (temperature $298 \mathrm{~K}$ and $1 \mathrm{~atm}$ of pressure), which were 20 and $10 \mathrm{~ns}$ for PT6-15c5e and PT1-15c5e, respectively, were conducted for each system and each metallic ion. Subsequently, all these simulations were repeated using identical conditions but without including the $\mathrm{PF}_{6}^{-}$counterions in the simulation boxes. It should be remarked that for all simulations the starting configurations were generated by accommodating the alkaline cations in the cavity of the macrocycles (i.e., one cation per one receptor).

Molecular Dynamics Simulations. Simulations were performed using the NAMD 2.6 program, $^{21}$ interactions being described using the potential energy functions of the AMBER force-field. ${ }^{22}$ Bond lengths involving hydrogen atoms were kept at their equilibrium distances using the SHAKE algorithm. ${ }^{23}$ Atom pair distance cutoffs were applied at $14.0 \AA$ to compute the van der Waals interactions. For the electrically neutral systems, electrostatic interactions were extensively computed by means of Ewald summations. The real space term was defined by the van der Waals cutoff, while the reciprocal space was computed by interpolation into an infinite grid of points (particle mesh Ewald) with maximum space grid points being $1.0 \AA^{24}$ The integration step was $2 \mathrm{fs}$ in all simulations.

Bond length and angles of the thiophene rings were kept fixed at the values provided by quantum mechanical calculations, ${ }^{25}$ whereas the 15 -crown-5-ether macrocycles and solvent molecules were not restrained. Inter-ring dihedral angles between consecutive thiophene rings $(\theta)$ were fixed at the values reported in previous works (i.e., $\left.\theta=-175^{\circ}\right){ }^{20,25}$ Specifically, B3LYP/6-31G(d) calculations were used to show that, independently of the arrangement adopted by the macrocycle, the all-trans is the most stable conformation for the polythiophene chain. Figure 1a displays both the

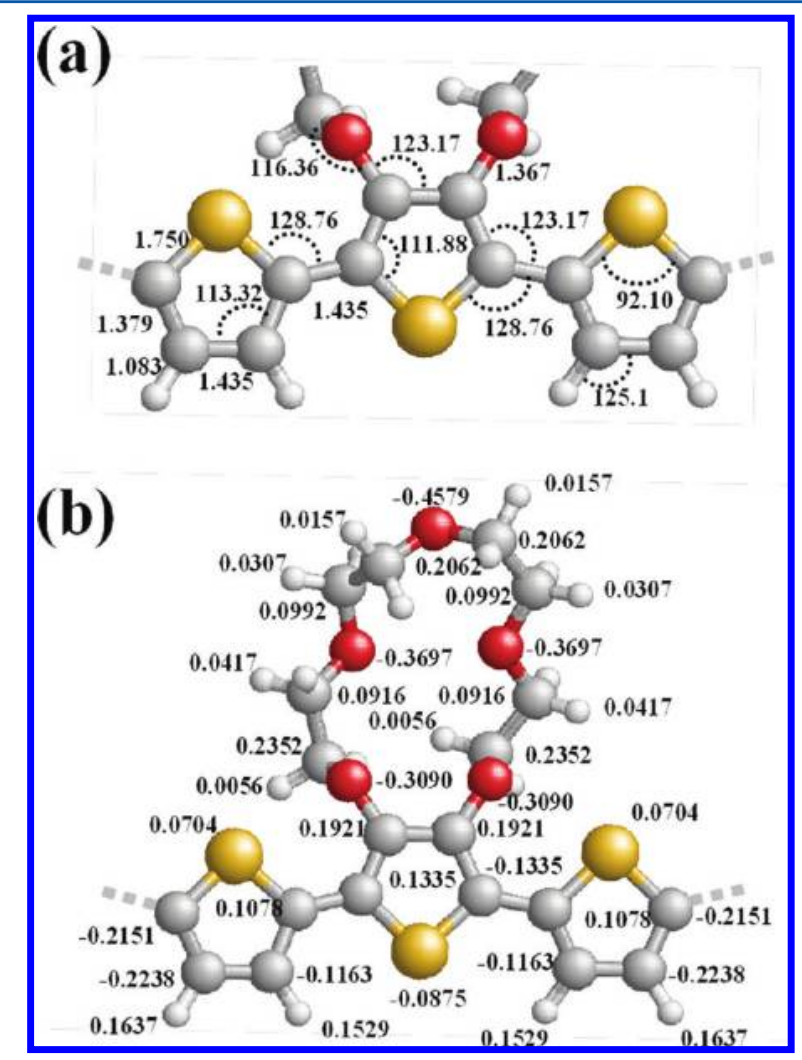

Figure 1. (a) Equilibrium bond lengths (in $\AA$ ) and bond angles (in degrees) used to describe the PTh segment. (b) Electrostatic parameters for the repeating unit of $\mathrm{PT} n-15 \mathrm{c} 5 \mathrm{e}$.

equilibrium bond lengths and equilibrium bond angles used in this work to describe the molecular geometry of the thiophene rings. Improper dihedral angles associated with the hydrogen atoms of thiophene rings were fixed at $180^{\circ}$. Stretching, bending and torsional parameters for the 15crown-5-ether macrocycle, as well as the van der Waals parameters for the all atoms involved in PTn-15c5e chains, were extrapolated from the standard AMBER99 libraries. ${ }^{22} \mathrm{~A}$ set of partial charges was explicitly derived for the repeating unit of PT $n-15 c 5 e$. Partial charges were obtained by fitting the 
rigorously defined quantum mechanical molecular electrostatic potential (MEP) to the Coulombic electrostatic potential. For consistency with the AMBER force field, the MEP was computed at the HF/6-31G(d) level. ${ }^{22}$ Atomic electrostatic parameters were derived by fitting the quantum mechanical MEP values into partial charges centered in the nuclei through a Levenberg-Marquardt nonlinear optimization procedure. Charges were calculated using different molecular geometries of PT1-15c5e that were derived from geometry optimizations in absence of ions. These geometries, which essentially differ in the arrangement of the macrocycle, led to similar charges. This feature is fully consistent with previously reported results, ${ }^{25}$ which indicated that the arrangement of the macrocycle does not dominate the conformational and electronic characteristics of crown ether functionalized PThs. Figure $1 \mathrm{~b}$ displays the electrostatic parameters obtained with this procedure and used for the MD simulations reported in this work. Finally, the solvent structure was described by using the dichloromethane all-atom model of the AMBER libraries.

Systems were relaxed to the experimental density value by performing new NPT-MD rounds with the solute frozen (Belly conditions). Before run $\mathrm{MD}$ trajectories, each simulation box was equilibrated as a whole system (i.e., solute plus solvent molecules, using the following strategy). Before any MD trajectory was run, $5 \times 10^{3}$ steps of energy minimization were performed in order to relax conformational and structural tensions. Next, different consecutive rounds of short MD runs were performed in order to equilibrate the density, temperature, and pressure. First, solvent and anions were thermally relaxed by three consecutives runs, while the polymer and alkaline cations were kept frozen: $0.5 \mathrm{~ns}$ of NVT-MD at $500 \mathrm{~K}$ were used to homogeneously distribute the solvent and anions in the box. After this, $0.5 \mathrm{~ns}$ of isothermal and $0.5 \mathrm{~ns}$ isobaric relaxation were run. Finally, all the atoms of the system were submitted to $0.15 \mathrm{~ns}$ of steady heating until the target temperature was reached $(298 \mathrm{~K}), 0.25 \mathrm{~ns}$ of $N V T-M D$ at $298 \mathrm{~K}$ (thermal equilibration) followed by $0.5 \mathrm{~ns}$ of density relaxation (NPT-MD). Both temperature and pressure were controlled by the weak coupling method, the Berendsen thermobarostat. ${ }^{26}$ The relaxation times used for the coupling were 1 and 10 ps for temperature and pressure, respectively. The end of the density relaxation simulation was the starting point of the molecular simulations presented in this work. All simulations were performed at $298 \mathrm{~K}$ and constant pressure of $1 \mathrm{~atm}$. The coordinates of all the production runs were saved every 500 steps ( 1 ps intervals).

Molecular Electrostatic Potential Maps. Interaction maps were obtained by calculating classically the MEP in a large set of points (see next section) placed outside the nuclear region, as stated by eq 1 :

$$
V\left(r_{1}\right)=\sum_{B} \frac{Q_{B}}{\left|r_{1}-R_{B}\right|}
$$

where $\left\{Q_{B}\right\}$ is the set of point charges defining the molecule charge distribution in force-field calculations.

Quantum Mechanical Calculations. Geometry optimizations of complexes formed by a PT1-15c5e molecule and an alkali metal ion $\mathrm{M}^{+}$(with $\mathrm{M}=\mathrm{Li}, \mathrm{Na}$, and $\mathrm{K}$ ) were performed in the gas-phase at the B3LYP level ${ }^{27,28}$ with the $6-31+G(d, p)$ basis set. ${ }^{29}$ Two starting geometries were considered for each complex. In the first $\mathrm{M}^{+}$was accommodated within the cavity defined by the macrocycle while in the second the alkaline cation was located at the external side of the macrocycle. These two positions are depicted in Scheme 2. Frequency calculations

Scheme 2. Position of the Alkaline Cations (Black Dots) in the Starting Geometries of PT1-15c5e $\cdots \mathrm{M}^{+}$Complexes

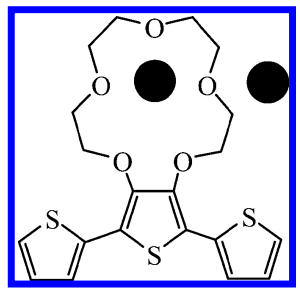

were carried out to verify the minimum energy state of all the optimized geometries. All the calculations were performed using the Gaussian $03^{30}$ computer program.

\section{RESULTS AND DISCUSSION}

Identification of the Binding Sites. Analysis of the last half of each trajectory indicates that, in all cases, alkaline ions are preferentially located out of the cavity of the crown ether substituent. Thus, although all alkaline ions were initially introduced at the center of such cavities, many of them tend to abandon such ideal positions after a few picoseconds of MD. Thus, they remain around the oxygen atoms of the macrocycle, occupying the cavity sporadically only. This is reflected in Figure 2, which represents the positions occupied by the $\mathrm{Li}^{+}$, $\mathrm{Na}^{+}$, and $\mathrm{K}^{+}$ions located around the PT6-15c5e molecule during the last $1 \mathrm{~ns}$ of $\mathrm{MD}$ simulations. As it can be seen, the functionalized PTh does not interact with all alkaline cations but with an average of $5\left(\mathrm{Li}^{+}\right), 3\left(\mathrm{Na}^{+}\right)$, and $6\left(\mathrm{~K}^{+}\right)$for simulations including $\mathrm{PF}_{6}^{-}$, and of 3 for simulations without

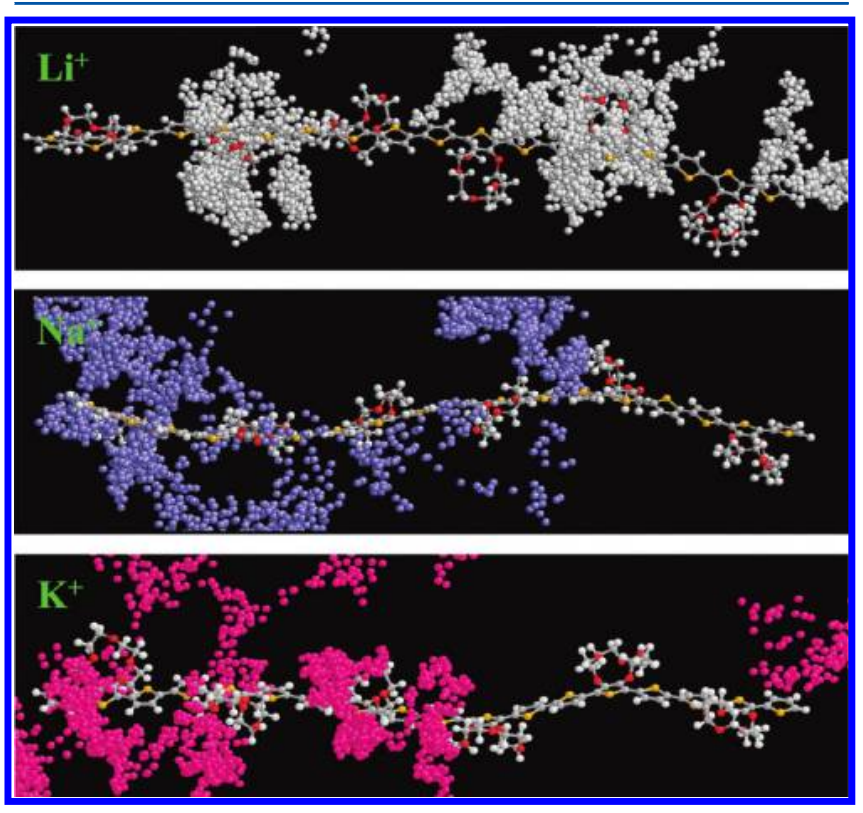

Figure 2. Schematic representation displaying the positions visted by the $\mathrm{Li}^{+}$(top), $\mathrm{Na}^{+}$(middle), and $\mathrm{K}^{+}$(bottom) ions at the last $\mathrm{ns}$ of $\mathrm{MD}$ simulations of PT6-15c5e with alkaline ions and $\mathrm{PF}_{6}{ }^{-}$ counterions. The positions are referred to the average conformation of PT6-15c5e. It should be noted that the conformation of the polythiophene backbone was kept fixed during the whole simulation, the only macrocycles being the only mobile groups of PT6-15c5e (see Methods). 
counterions, independently of the alkaline cation. Another important trend is that, among the cations that occupy the cavity of the crown ether, the $\mathrm{Na}^{+}$is the one with highest degree of confinement (Figure 3). Thus, the size of this cation

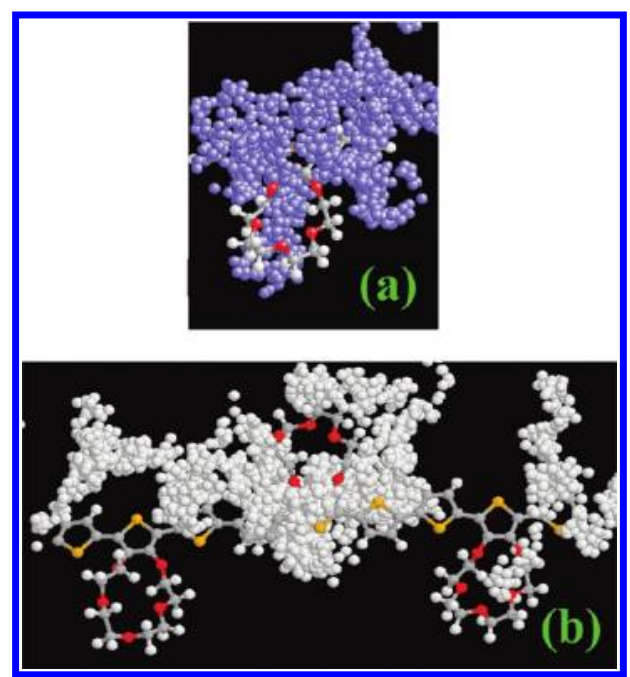

Figure 3. Details (magnification) of the (a) $\mathrm{Na}^{+}$and (b) $\mathrm{Li}^{+}$ions visiting the cavity of the macrocycle. One and three repeating units are displayed in parts a and b, respectively. As mentioned in the Methods, the conformation of the polythiophene backbone was kept fixed during the whole simulation, the only macrocycles being the only mobile groups of PT6-15c5e.

is suitable to fit within the receptor. ${ }^{20}$ In contrast, the small size of $\mathrm{Li}^{+}$facilitates its mobility around the macrocycle, which is induced by the thermal energy (Figure 2).

In order to explain the fact that the region around the crown ether is more visited by alkaline cations than the cavity, MEP maps were computed using the classical expression displayed in eq 1. According to this expression, the MEP represents the interaction of crown ether functionalized PTh with a positive charge. The MEP was evaluated over a grid of $60 \times 60 \times 60 \AA^{3}$ constructed around a PT6-15c5e model molecule using a grid width of $0.5 \AA$. Figure $4 \mathrm{a}$ shows the regions with lower MEP values (i.e., MEP $<-10 \mathrm{kcal} / \mathrm{mol}$ ) for a fragment with two repeating units. These attractive regions, which should be considered as the preferred binding sites of alkaline cations, are located at: the external side of the macrocycles, the cavity of the macrocycles (i.e., a small region close to the oxygen atoms), and the nonfunctionalized thiophene rings (i.e., above and below the unsubstituted thiophene rings). Obviously, the preference by these regions is due to the role played by the oxygen atoms of the receptors and the $\pi$ electron density of the aromatic rings, respectively. As it can be seen, the attractive MEP regions displayed in Figure $4 \mathrm{a}$ are fully consistent with the positions occupied by alkaline cations in Figures 2 and 3 .

On the other hand, Figure $4 \mathrm{~b}$ displays the regions with higher MEP values (i.e., MEP > $+10 \mathrm{kcal} / \mathrm{mol}$ ), while the superposition of the attractive and repulsive regions is represented in Figure 4c. The repulsive regions are essentially located above and below the receptor, indicating that cations need to overcome a repulsive region to enter into the cavity of the crown ether. It should be remarked that these results are independent of the polymer conformation. This is reflected in Figure $4 \mathrm{~d}$, which shows the superposition of the regions with

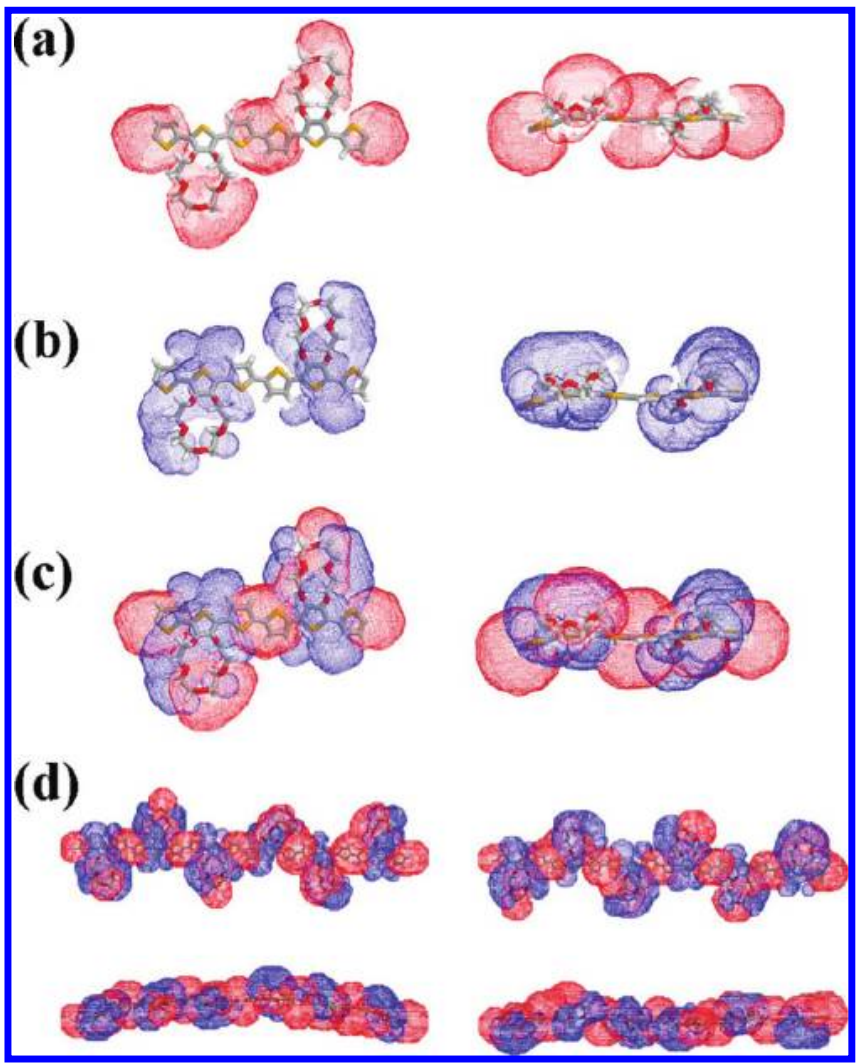

Figure 4. Molecular electrostatic potential (MEP) represented around two repeating units of the crown ether functionalized polythiophene. Representations correspond to the equatorial (left) and axial (right) projections of regions with (a) MEP values lower than $-10 \mathrm{kcal} / \mathrm{mol}$ and (b) MEP values higher than $+10 \mathrm{kcal} / \mathrm{mol}$, (c) the superposition of the attractive and repulsive regions displayed in parts $a$ and $b$, and (d) superposition of attractive and repulsive regions for two different conformation of PT6-15c5e. The MEP was calculated over a grid of 60 $\times 60 \times 60 \AA^{3}$ with a grid width of $0.5 \AA$.

lower and higher MEP for two conformers of PT6-15c5e extracted from different MD simulations.

Dynamics of the Binding Sites. Figure 5 represents the temporal evolution of the distance between each alkaline ion and the center of masses of the closest cavity receptor for the simulations performed considering a chain of PT6-15c5e. As it can be seen, the information provided by simulations with and without $\mathrm{PF}_{6}^{-}$is significantly different. Thus, four different situations, which are depicted in Scheme 3, were identified in the previous subsection for the alkaline ions: (1) the cation is accommodated in the cavity of the receptor; (2) the cation interacts with the nonfunctionalized thiophene rings; (3) the cation interacts with the external side of the macrocycle, remaining in a position relatively close to the PTh backbone, i.e., the average distance to the PTh backbone is $\sim 3.5-4.0 \AA$; and (4) cations are solvated far away from the polymer chain, i.e., the average distance to the polymer chain is larger than 10 $\AA$. Table 1 lists the average distribution of the cations among the four possible arrangements during the simulations and for category 1 , the average distance between the cation and the center of masses of the closest crown ether.

The number of $\mathrm{Li}^{+}, \mathrm{Na}^{+}$, and $\mathrm{K}^{+}$ions contained in the receptors of PT6-15c5e (distances lower than $1.5 \AA$ in Figure 5) is 1,1 and 2, respectively, for simulations with $\mathrm{PF}_{6}^{-}$, and 2, 2 , and 3 for the simulations without counterions. Thus, the 

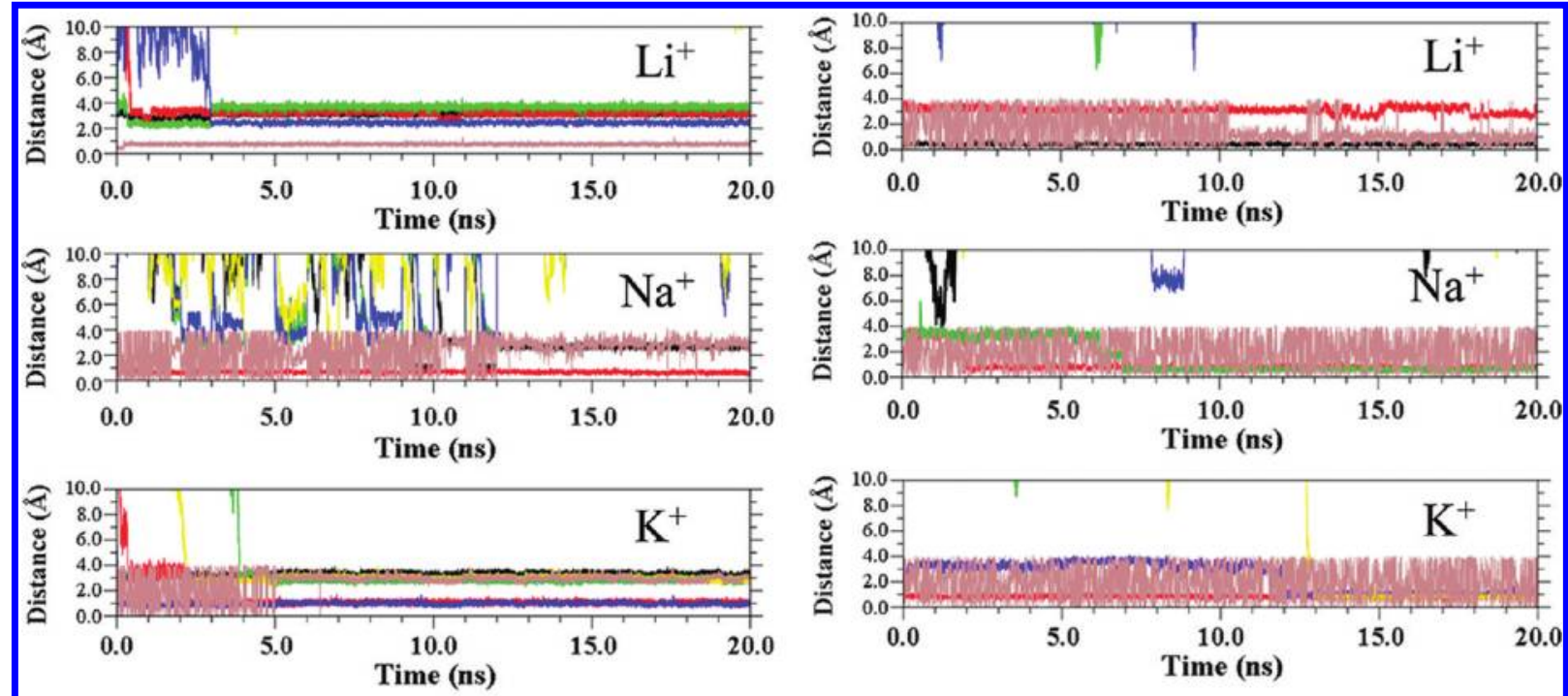

(a)

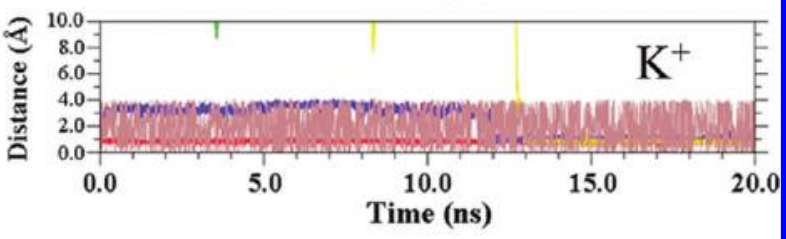

(b)

Figure 5. Temporal evolution of the distance between each of the six alkaline cations and the center of masses of the closest crown ether. Distances obtained for both simulations on PT6-15c5e with (a) and without (b) considering $\mathrm{PF}_{6}{ }^{-}$counterions are displayed.

Scheme 3. Arrangements Found for the Alkaline Ions During the MD Simulations

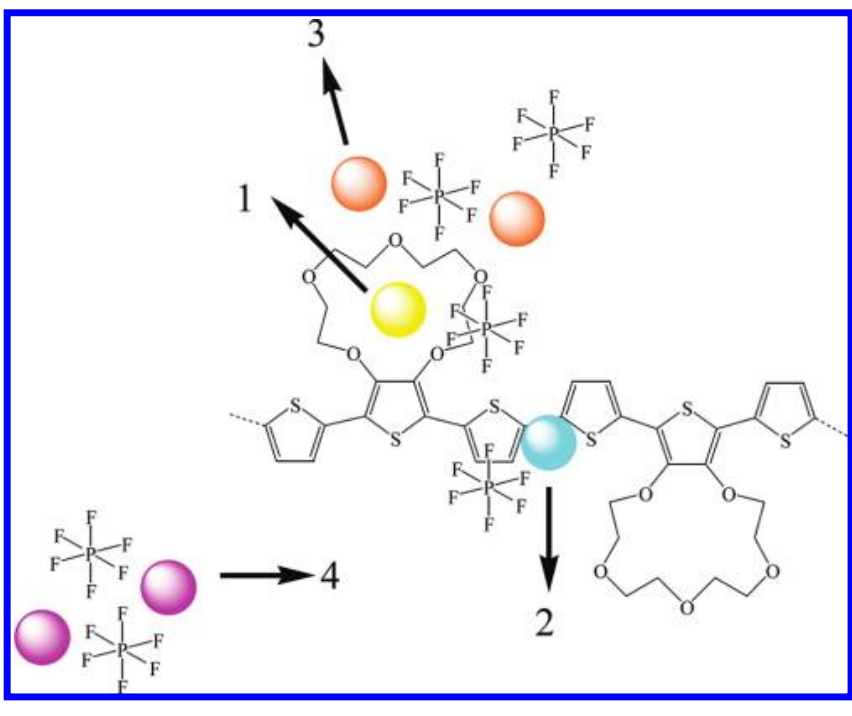

Table 1. Distribution of the Alkaline Cations among the Four Categories Displayed in Scheme 3 (See Text) During the MD Simulations of PT6-15c5e $\mathrm{e}^{a}$

\begin{tabular}{ccccc} 
& \multicolumn{4}{c}{ simulation } \\
\cline { 2 - 5 } & 1 & 2 & 3 & 4 \\
PT6-15c5e $\cdots 6 \mathrm{Li}^{+} \cdot 6 \mathrm{PF}_{6}^{-}$ & $1(1.865 \pm 0.203)$ & 1 & 4 & 0 \\
PT6-15c5e $\cdots 6 \mathrm{Li}^{+}$ & $2(2.477 \pm 2.334)$ & 1 & 0 & 3 \\
PT6-15c5e $\cdots 6 \mathrm{Na}^{+} \cdot 6 \mathrm{PF}_{6}^{-}$ & $1(1.674 \pm 0.190)$ & 1 & 1 & 3 \\
PT6-15c5e $\cdots 6 \mathrm{Na}^{+}$ & $2(1.745 \pm 0.229)$ & 0 & 0 & 4 \\
PT6-15c5e $\cdots 6 \mathrm{~K}^{+} \cdot 6 \mathrm{PF}_{6}^{-}$ & $2(2.493 \pm 0.302)$ & 1 & 3 & 0 \\
PT6-15c5e $\cdots 6 \mathrm{~K}^{+}$ & $3(2.226 \pm 0.295)$ & 0 & 0 & 3
\end{tabular}

${ }^{a}$ For category 1, the average distance and the standard error (in $\AA$ ) between the cation and the center of masses of the closest macrocycle is included. absence of counterions seems to affect the mobility of the metallic ions, reducing it. However, the most remarkable feature is that, although in the starting arrangements the six macrocycles were occupied by the alkaline cations, after a few picoseconds some of them became empty. Moreover, when arrangement 1 involves 2 or 3 ions, they tend to occupy the cavities of nonconsecutive of macrocycles suggesting that electrostatic repulsions induce the escape of cations at the beginning of the simulations. Another important difference between the two kinds of simulations refers to arrangements 3 and 4 . More specifically, arrangement 3 is only reachable when the alkaline cation is associated with the counterion, cations evolving rapidly toward arrangement 4 in the simulations without $\mathrm{PF}_{6}{ }^{-}$anions. This feature is consistent with the MEP maps displayed in Figure 4. Thus, attractive electrostatic regions were identified at the side of the receptors while repulsive electrostatic regions were found above and below the macrocycles, the latter being suitable to accommodate the negatively charged counterions. Accordingly, a strong electrostatic pair is formed when the alkaline cation and the counterion are located at such attractive and repulsive zones, respectively.

Solvation of Alkaline Ions and Formation of Ion-Pairs. The solvation of crown ethers and their complexes with metallic cations in water and different ionic liquids has been investigated using MD simulations by Wipff and co-workers. ${ }^{31-36}$ Thus, polarity of water and the presence of solvent ions play a crucial role in the ability and efficiency of simple crown ether molecules (i.e., macrocycles not fused to a polymer chains) to extract alkali and alkaline cations. ${ }^{37-40}$ However, simulations reported in this work were carried out in dichloromethane solution because of the PThs functionalized with crown ether macrocycles are not soluble in water or any other polar solvent. Therefore, examination of the effects produced by this organic solvent on the systems under study is necessary.

Dichloromethane molecules only form weak interactions with $\mathrm{Li}^{+}, \mathrm{Na}^{+}$, and $\mathrm{K}^{+}$and, therefore, no clear coordination nor well-defined solvation shell were identified around these 
metallic ions. These features are reflected in Table 2, which shows the lowest and highest values of both the average

Table 2. Average Number of Dichloromethane Molecules around the Alkaline Ions $\left(n_{s}\right)$, Average Time in Which the Solvent Molecules Are Around the Cation $\left(\tau_{s}\right.$, in ps), Number of Cations Involved in the Formation of Ion-Pairs $\left(n_{c}\right)$, and the Interval Defined by the Lowest and Highest Number of Different Ion-Pairs Formed by the Cations of Each Species $\left(n_{a}\right)$

\begin{tabular}{lllll}
\multicolumn{1}{c}{ simulation } & \multicolumn{1}{c}{$n_{s}{ }^{a}$} & \multicolumn{1}{c}{$\tau_{s}^{b}$} & $n_{c}{ }^{c}$ & $n_{a}$ \\
PT6-15c5e $\cdots 6 \mathrm{Li}^{+} \cdot 6 \mathrm{PF}_{6}{ }^{-}$ & $5.6-5.9$ & $30-103$ & 6 & $1-4$ \\
PT6-15c5e $\cdots 6 \mathrm{Li}^{+}$ & $6.9-7.6$ & $9-150$ & - & - \\
PT6-15c5e $\cdots 6 \mathrm{Na}^{+} \cdot 6 \mathrm{PF}_{6}^{-}$ & $5.8-6.0$ & $15-112$ & 5 & $2-3$ \\
PT6-15c5e $\cdots 6 \mathrm{Na}^{+}$ & $5.0-6.0$ & $8-78$ & - & - \\
PT6-15c5e $\cdots 6 \mathrm{~K}^{+} \cdot 6 \mathrm{PF}_{6}^{-}$ & $5.6-6.2$ & $20-95$ & 5 & $2-4$ \\
PT6-15c5e $\cdots 6 \mathrm{~K}^{+}$ & $5.5-5.9$ & $9-127$ & - & -
\end{tabular}

${ }^{a}$ Solvent molecules with at least one atom at a distance lower than $4.50 \AA$ from the alkali cation are included in $n_{c}$. ${ }^{b}$ Residence time (in ps) within the cutoff sphere used to define $n_{c}$. ${ }^{c}$ An ion-pair is considered as formed when the interaction between the cation and the anion remains by at least $10 \mathrm{ps}$.

number of solvent molecules around the alkaline ions $\left(n_{s}\right)$ and the average time in which each of such nearest solvent molecules was continuously within such cutoff $\left(\tau_{s}\right)$. The values of $n_{s}$ were determined by considering at least one atom of the solvent molecule within the cutoff distance. As it can be seen, the $n_{s}$ values obtained for the three types of metallic ions were very similar for simulations with $\mathrm{PF}_{6}{ }^{-}$counterions, ranging from 5.6 to 6.0. Moreover, these values are fully consistent with those derived from simulations without counterions for $\mathrm{Na}^{+}$ and $\mathrm{K}^{+}$, even though $n_{s}$ increases to 6.9-7.6 in the case of $\mathrm{Li}^{+}$. On the other hand, $\tau_{s}$ ranges from 8 to 127 ps indicating a very rapid exchange with molecules of the bulk.

Table 2 also lists the values of $n_{c}$ and $n_{a}$ for the simulations with $\mathrm{PF}_{6}{ }^{-}$counterions, which refer to the number of cations of a given type forming ion-pairs during at least $10 \mathrm{ps}$ and the interval defined the lowest and highest number of $\mathrm{PF}_{6}{ }^{-}$that interact with the cations included in $n_{\mathcal{c}}$, respectively. As it can be seen, all (the six $\mathrm{Li}^{+}$) or almost all (five $\mathrm{Na}^{+}$and five $\mathrm{K}^{+}$) cations participated in the formation of ion-pairs. Moreover, each cation interacted with more than one $\mathrm{PF}_{6}{ }^{-}$counterion, the only exception to this behavior being one $\mathrm{Li}^{+}$that was exclusively involved in one ion-pair. Indeed, $2 \mathrm{Li}^{+}, 3 \mathrm{Na}^{+}$, and $4 \mathrm{~K}^{+}$ participated in at least three ions pairs along the whole trajectory. The lifetime of the ion-pairs ranged from 190 to 2.3 ns, the average value being 674 ps.

Entropic Analysis of the Binding Process. The relative entropy $(\Delta S)$ calculated for the "free state $\rightarrow$ bound state" process was estimated using the method originally developed by Schlitter ${ }^{41}$ and latter improved by van Gunsteren and coworkers, ${ }^{42}$ which is based on a covariance matrix atompositional fluctuations. The entropy of the free-state was derived from additional simulations on the $\mathrm{M}^{+}: \mathrm{PF}_{6}^{-}$complexes and isolated $\mathrm{M}^{+}$in dichloromethane solution, where $\mathrm{M}^{+}$refers to $\mathrm{Li}^{+}, \mathrm{Na}^{+}$, and $\mathrm{K}^{+}$. As the interactions between dichloromethane and the rest of chemical species are relatively weak with respect to those expected for other polar solvents (e.g., water), the entropic contribution of this organic solvent to the free and bound states have been considered to be similar (i.e., the two contributions cancel in the whole process). A similar approach has been considered for the $\mathrm{PF}_{6}{ }^{-}$counterions, which only interact with the solvent and the metallic ions. The temporal evolution of the accumulative average entropy calculated for each of the six individual ions in $\mathrm{PF}_{6}^{-}$-containing systems is depicted in Figure 6a. As it can be seen, in all three cases the six ions converge to similar values, the averaged $\Delta S$ values for the $\mathrm{Li}^{+}, \mathrm{Na}^{+}$, and $\mathrm{K}^{+}$cations accommodated in the cavity of the crown ether (case 1 in Table 1) being 8.2, 30.6, and $22.1 \mathrm{cal} / \mathrm{mol} \cdot \mathrm{K}$. The $-\mathrm{T} \Delta S$ values estimated for the formation of complexes with the receptors of PTn-15c5e at 298 $\mathrm{K}$ are $-2.4,-9.1$, and $-6.5 \mathrm{kcal} / \mathrm{mol}$, which agree with the experimental observation that the affinity toward $\mathrm{Na}^{+}$is higher than toward $\mathrm{Li}^{+}$and $\mathrm{K}^{+} .8,9$ Moreover, the entropic contributions are consistent with the average distance between the cation and the center of masses of the macrocycle (Table 1).

The entropies of the alkaline ions derived from the trajectories without $\mathrm{PF}_{6}^{-}$are displayed in Figure $6 \mathrm{~b}$. The

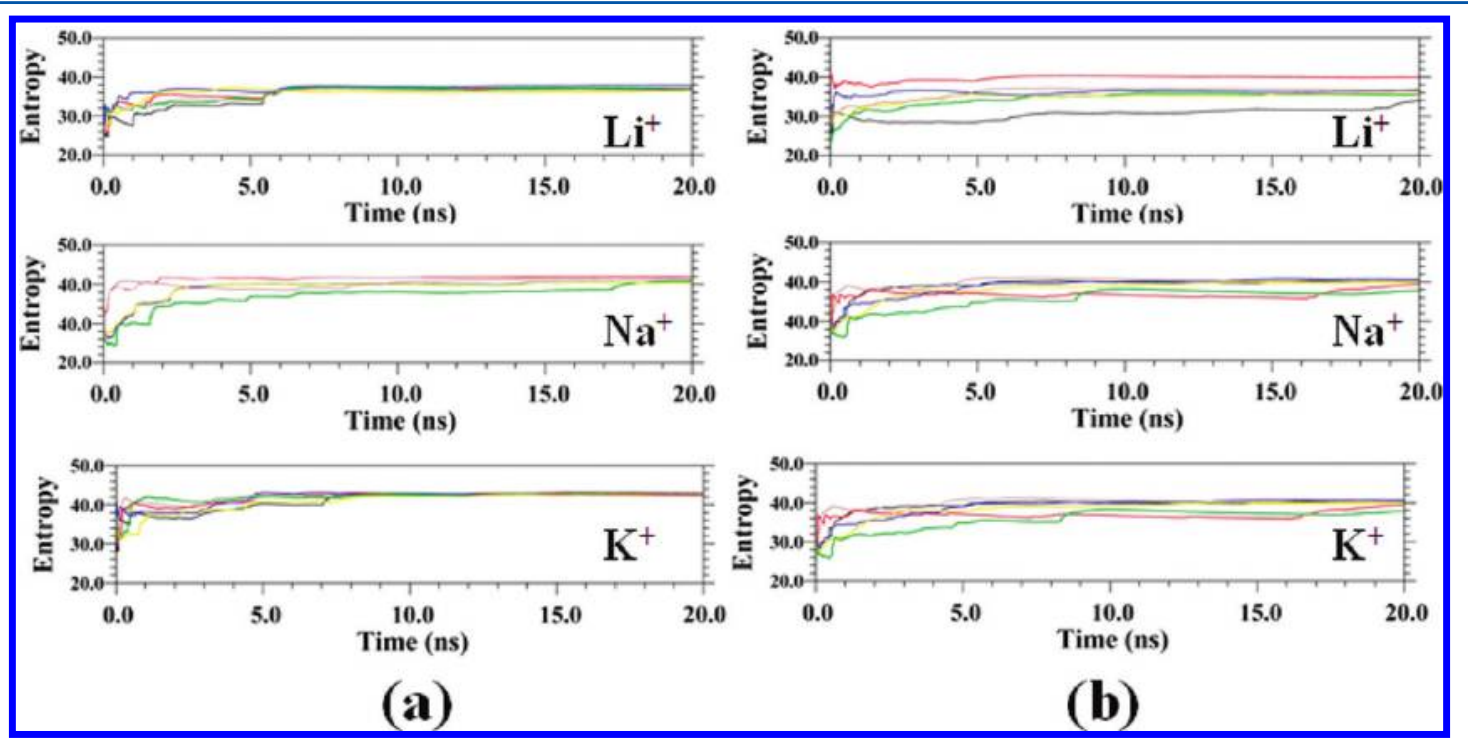

Figure 6. Temporal evolution of the average entropy (in cal/K·mol) for the alkaline cations derived from MD simulations on PT6-15c5e with (a) and without $(\mathrm{b})$ considering $\mathrm{PF}_{6}^{-}$counterions. It should be noted that the evolution averages, which are accumulative, clearly converge in all cases. 
convergence of cations with different arrangements is considerably slower than in Figure 6a. In spite of this, the averaged $\Delta S$ values for the alkaline cations accommodated in the cavity of the crown ether are similar to those obtained for $\mathrm{PF}_{6}{ }^{-}$-containing simulations, i.e. $8.6,28.6$, and $20.5 \mathrm{cal} / \mathrm{K} \cdot \mathrm{mol}$ for $\mathrm{Li}^{+}, \mathrm{Na}^{+}$, and $\mathrm{K}^{+}$, respectively.

Figure 7 represents the temporal evolution of the distance between the alkaline ion and the center of masses of the cavity

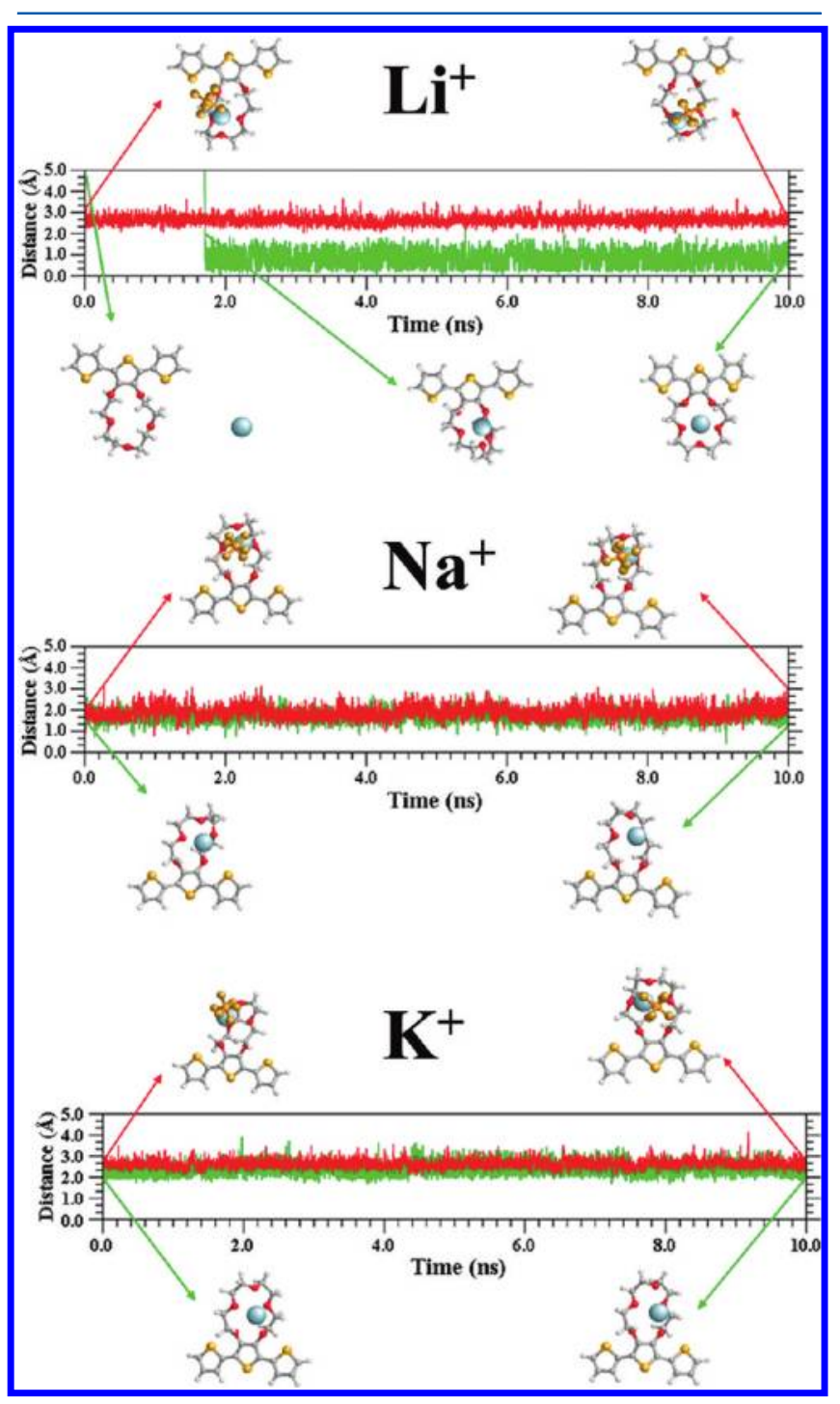

Figure 7. Temporal evolution in MD simulations on PT1-15c5e with (red) and without (green) considering the $\mathrm{PF}_{6}{ }^{-}$counterion of the distance between the alkaline cation and the center of masses of the crown ether.

for the simulations on PT1-15c5e. The alkaline ion was inside the cavity of the receptor during the whole trajectory in all cases with exception of one $\mathrm{Li}^{+}$. Thus, although the results with $\mathrm{Na}^{+}$ and $\mathrm{K}^{+}$do not depend on the counterion, $\mathrm{PF}_{6}^{-}$affects significantly the behavior of $\mathrm{Li}^{+}$. More specifically, significant fluctuations around its equilibrium position, which is located very close to the center of masses of the macrocycle, are produced after $1.9 \mathrm{~ns}$ by the absence of $\mathrm{PF}_{6}{ }^{-}$. Both fluctuations and proximity to the center of masses were possible because, in this case, the crown ether tends to adopt a planar arrangement. On the other hand, the entropy derived for $\mathrm{Li}^{+}, \mathrm{Na}^{+}$, and $\mathrm{K}^{+}$ using the data of the $\mathrm{PF}_{6}{ }^{-}$-containing trajectory is $10.5,18.3$, and $-3.7 \mathrm{cal} / \mathrm{K} \cdot \mathrm{mol}$, respectively, i.e., $-T \Delta S=-3.1,-5.4$ and $+1.1 \mathrm{kcal} / \mathrm{mol}$, whereas the value in absence of counteanion is $-3.7,17.8$, and $11.4 \mathrm{cal} / \mathrm{K} \cdot \mathrm{mol}$, i.e., $-T \Delta S=+1.1,-5.3$, and $-3.4 \mathrm{kcal} / \mathrm{mol}$. These values are in qualitative agreement with those obtained for PT6-15c5e.

Comparison of the experimental measures for the binding process, which were expressed as free energies, ${ }^{9,10}$ with both the enthalpies reported in our previous study ${ }^{20}$ and the relative entropies calculated in this work confirm that the binding of PTn-15c5e with alkali ion is an entropically driven process. Thus, previous QM calculations predicted that the enthalphy of binding becomes more favorable when as the size of the ion decreases, while the entropic contribution is more favorable for $\mathrm{Na}^{+}$than for $\mathrm{Li}^{+}$and $\mathrm{K}^{+}$. Indeed, despite of the number of favorable binding sites for alkaline cations, the selectivity of PT $n-15 \mathrm{c} 5 \mathrm{e}$ toward $\mathrm{Na}^{+}$is explained by the capability of this ion to accommodate within cavity at the electrostatically attractive binding site.

Quantum Mechanical Calculations. The interaction of alkaline cations $\left(\mathrm{Li}^{+}, \mathrm{Na}^{+}\right.$, and $\left.\mathrm{K}^{+}\right)$with the $\pi$ electron density of the thiophene ring was studied in recent works using different DFT functionals and ab initio (MP2 and MP4) methods combined with the $6-31+G(d, p)$ basis set. ${ }^{43,44}$ In particular, it was found that these complexes, which are dominated by a strong electrostatic interaction between the $\pi$ cloud of the heterocycle and the positively charged the alkaline atom, are not only stable (i.e., interaction energies were found to be $-31.2,-19.6$, and $-14.0 \mathrm{kcal} / \mathrm{mol}$ for $\mathrm{Li}^{+}, \mathrm{Na}^{+}$, and $\mathrm{K}^{+}$, respectively ${ }^{43}$ ) but also minimum energy states. The stability of these electrostatic charge transfer complexes is in excellent with agreement with the $\mathrm{MD}$ results since, as evidenced in the previous sections, above and below the $\pi$-system of unsubstituted thiophene rings were identified as one of the preferred binding sites in PTn-15c5e.

The stability of the other two preferred binding sites, the cavity and the external side of the macrocycles, has been investigated in this work using quantum mechanical calculations. Specifically, two complexes involving a PT1-15c5e molecule interacting with a metallic ion were considered for each alkaline cation. These two complexes differ in the location of the ion, which has been accommodated in the cavity of the macrocycle and in the external side of the crown ether (see Scheme 2). The geometries of the six complexes (i.e., 2 arrangements for 3 different alkaline cations) were optimized at the B3LYP/6-31+G(d,p) level. Frequency calculations allowed us to confirm the nature of minimum state of the optimized geometries, which are displayed in Figure 8.

As it can be seen, the conformation of the macrocycle was similar in the three minima with the alkaline ion located at the external side of the macrocycle. However, the shape of the crown ether is clearly influenced by the size of the alkaline ion in the minima with the metallic cation accommodated in the cavity. More specifically, the $\mathrm{Na}^{+}$fits in the cavity of the crown ether, no conformational distortion being observed in this case. In contrast, $\mathrm{Li}^{+}$and $\mathrm{K}^{+}$are small and large with respect to the dimensions of the cavity and, therefore, optimization of the interactions involves alteration of the shape of the macrocycle. Analysis of the relative energies reveals that the minimum with the metallic ion located at the external side of the macrocycle is considerably less stable than that with the cation accommodated in the cavity (i.e., 23.7, 19.7, and $9.1 \mathrm{kcal} / \mathrm{mol}$ for $\mathrm{Li}^{+}$, $\mathrm{Na}^{+}$, and $\mathrm{K}^{+}$, respectively). 


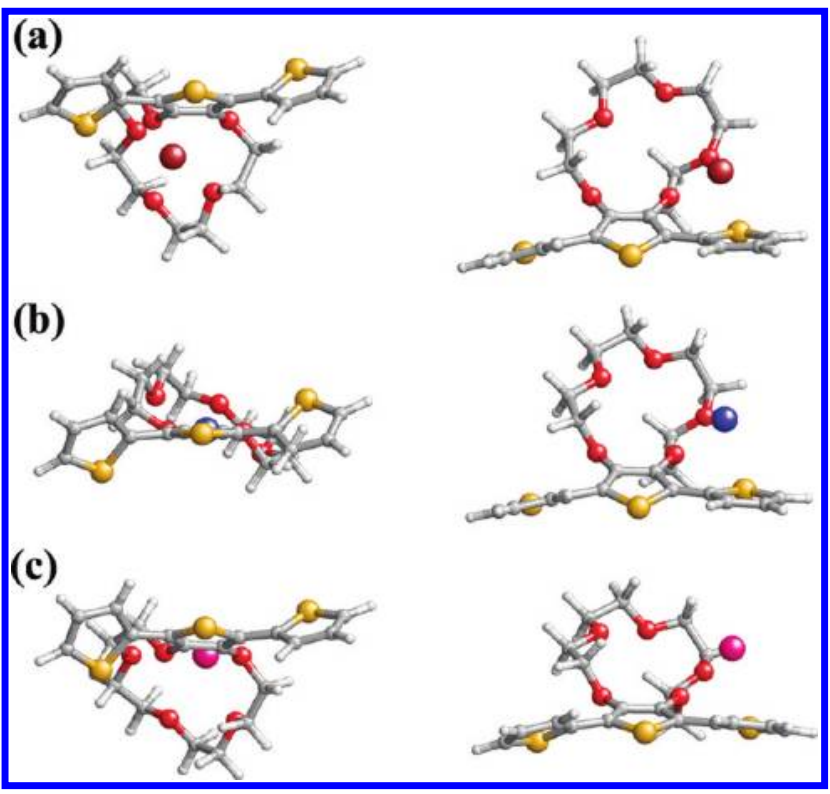

Figure 8. Minimum energy structures obtained for PT1-15c5e $\cdots \mathrm{M}^{+}$ complexes with $\mathrm{M}^{+}=$(a) $\mathrm{Li}^{+}$, (b) $\mathrm{Na}^{+}$, and (c) $\mathrm{K}^{+}$. Two minima are displayed for each alkaline ion: one with the cation accommodated in the cavity (left) and one with the cation at the external side of the macrocycle (right). The orientation used to draw each type of structure has been selected according to the features discussed in the text.

These, quantum mechanical calculations are fully consistent with MD results. Thus, classical MD simulations allowed us to identify three preferred binding sites, which have been also detected in the minimum energy complexes derived from DFT geometry optimizations. Moreover, in all cases the lowest energy minimum corresponds to the complex in which the ion is located at the cavity of the macrocycle. However, the size of this cavity is particularly appropriated for $\mathrm{Na}^{+}$, explaining the selectivity of this receptor. On the other hand, the five oxygen atoms of the crown ether generate a very large number of possible binding sites at the external side of the macrocycle. Accordingly, these kind of binding sites are entropically favored with respect to the cavity, which is much more confined. This feature is fully consistent with our MD observations in which the positions of the metallic cations are largely influenced by the kinetic energy and external forces associated with the counterions and solvent molecules.

\section{CONCLUSIONS}

Although MD simulations on a model system involving only one receptor provide a reliable estimation of the binding entropies, larger model molecules are necessary to describe completely the sensing response of PTn-15c5e. Thus, simulations on PT6-15c5e reflect that many alkaline cations escape from the cavities of the macrocycles at the beginning of the trajectory. This should be attributed to the electrostatic attraction exerted by and the large molecular regions with a high concentration of oxygen atoms and $\pi$-electron density, which are relatively close to the cavity. Thus, the external side of the macrocycles and the two faces (i.e., above and below) of the unsubstituted thiophene rings, together with the small and confined region of the cavity close to the oxygen atoms, are the preferred binding sites of alkaline cations from an electrostatic point view. The stability associated with all these binding sites has been corroborated by quantum mechanical calculations. We also observed that typically only the cavities of two nonconsecutive receptors remain filled after a few picoseconds.

The enthalpic order of affinity toward ions previously predicted by $\mathrm{MP} 2 / 6-31+\mathrm{G}(\mathrm{d}, \mathrm{p})$ calculations in the gasphase $^{20}$ was not consistent with the observation that $\mathrm{Na}^{+}$ presents the highest affinity. 8 ,9 However, the results reported in this work allow us to complete the scenario that explains the response of crown ether functionalized PThs to alkaline cations. Thus, in spite of the cavity is not the only binding site, the selectivity should be attributed to the ability of the cations to fit into the cavity, which in turn is consequence of their respective sizes. Accordingly, the selective preference toward a given alkaline ion grows with its degree of the confinement within the macrocycle, defining the entropic contribution of the binding process. The $-\mathrm{T} \Delta S$ values obtained for PT6-15c5e in chloroform solution $\left(-2.4,-9.1\right.$, and $-6.5 \mathrm{kcal} / \mathrm{mol}$ for $\mathrm{Li}^{+}$, $\mathrm{Na}^{+}$and $\mathrm{K}^{+}$, respectively) indicate that the affinity toward is consequence of an entropically driven effect. Finally, comparison between the results obtained in presence and absence of $\mathrm{PF}_{6}{ }^{-}$evidence quantitative differences, even though counterions are not essential to obtain a reasonable qualitative description of the mechanism.

\section{AUTHOR INFORMATION}

\section{Corresponding Author}

*E-mail: carlos.aleman@upc.edu.

\section{Notes}

The authors declare no competing financial interest.

¿Collaborateur Scientifique" of the Belgian National Fund for Scientific Research.

"Senior research associate of the FNRS.

\section{ACKNOWLEDGMENTS}

This work has been supported by MCYT and FEDER (MAT2009-09138), and by the Generalitat de Catalunya (research group 2009 SGR 925 and XRQTC). Authors are indebted to the Centre de Supercomputació de Catalunya (CESCA) for use of computational facilities. J.P. acknowledges the Belgian National Fund for Scientific Research for his grant as "Collaborateur Scientifique" (FNRS-FSR). Support for the research of C.A. was received through the prize "ICREA Academia” for excellence in research funded by the Generalitat de Catalunya.

\section{REFERENCES}

(1) McQuade, D. T.; Pullen, A. E.; Swager, T. M. Chem. Rev. 2000, 100, 2537.

(2) Goldenberg, L. M.; Bryce, M. R.; Petty, M. C. J. Mater. Chem. 1999, 9, 1957.

(3) Roncali, J. J. Mater. Chem. 1999, 9, 1875.

(4) Sone, T.; Sato, K.; Ohba, Y. Bull. Chem. Soc. Jpn. 1989, 62, 838.

(5) Parker, D.; Kataky, R.; Nicholson, P. E.; Covington, A. K. Analyst 1991, 116, 135.

(6) Tsukube, H. Talanta 1993, 40, 1313.

(7) Sannicolò, F.; Brenna, E.; Bennincori, T.; Zotti, G.; Zecchin, S.; Schiavon, G.; Pilati, T. Chem. Mater. 1998, 10, 2167.

(8) Bäuerle, P.; Scheib, S. Adv. Mater. 1993, 5, 848.

(9) Bäuerle, P.; Scheib, S. Acta Polym. 1995, 46, 124.

(10) Scheib, S.; Bäuerle, P. J. Mat Chem. 1999, 9, 2139.

(11) Rimmel, G.; Bäuerle, P. Synth. Met. 1999, 102, 1323.

(12) Bäuerle, P.; Götz, G.; Hiller, M.; Scheib, S.; Fischer, T.; Segelbacher, U.; Bennati, M.; Grupp, A.; Mehring, M.; Stoldt, M.; Seidel, C.; Geiger, F.; Schweizer, H.; Umbach, E.; Schmelzer, M.; Roth, 
S.; Egelhaaf, H. J.; Oelkrug, D.; Emele, P.; Port, H. Synth. Met. 1993, 61,71 .

(13) Marsella, M. J.; Swager, T. M. J. Am. Chem. Soc. 1993, 115, 12214.

(14) Marsella, M. J.; Swager, T. M. Adv. Mater. 1994, 6, 595.

(15) Swager, T. M. Acc. Chem. Res. 1998, 31, 201.

(16) Roncali, J.; Garreau, R.; Lemaire, M. J. Electroanal. Chem. 1990, 278, 373.

(17) Jousselme, B.; Blanchard, P.; Levillain, E.; Delaunav, J.; Allain, M.; Richomme, P.; Rondeau, D.; Gallego-Planas, N.; Roncali, J. J. Am. Chem. Soc. 2003, 125, 1364.

(18) Demeter, D.; Blanchard, P.; Allain, M.; Grosu, I.; Roncali, J. J. Org. Chem. 2007, 72, 5285.

(19) Demeter, D.; Blanchard, P.; Grosu, I.; Roncali, J. Electrochem. Commun. 2007, 9, 1587.

(20) Casanovas, J.; Preat, J.; Zanuy, D.; Alemán, C. Chem.-Eur. J. 2009, 15, 4676.

(21) Phillips, J. C.; Braun, R.; Wang, W.; Gumbart, J.; Tajkhorshid, E.; Villa, E.; Chipot, C.; Skeel, R. D.; Kale, L.; Schulten, K. J. Comput. Chem. 2005, 26, 1781.

(22) Cornell, W. D.; Cieplak, P.; Bayly, C. I.; Gould, I. R.; Merz, K. M., Jr.; Ferguson, D. M.; Spellmeyer, D. C.; Fox, T.; Caldwell, J. W.; Kollman, P. A. J. Am. Chem. Soc. 1995, 117, 5179.

(23) Ryckaert, J. P.; Ciccotti, G.; Berendsen, H. J. C. J. Comput. Phys. 1977, 23, 327.

(24) Darden, T.; York, D.; Pedersen, L. J. Chem. Phys. 1993, 98, 10089.

(25) Casanovas, J.; Alemán, C. Macromolecules 2008, 41, 3919.

(26) Berendsen, H. J. C.; Postma, J. P. M.; van Gunsteren, W. F.;

DiNola, A.; Haak, J. R. J. Chem. Phys. 1984, 81, 3684.

(27) Becke, A. D. J. Chem. Phys. 1993, 98, 1372.

(28) Lee, C.; Yang, W.; Parr, R. G. Phys. Rev. B 1993, 37, 785.

(29) McLean, A. D.; Chandler, G. S. J. Chem. Phys. 1980, 72, 5639.

(30) Gaussian 03, Revision B.02, Frisch, M. J.; Trucks, G. W.; Schlegel, H. B.; Scuseria, G. E.; Robb, M. A.; Cheeseman, J. R.; Montgomery, J. A.; Vreven, T.; Jr., Kudin, K. N.; Burant, J. C.; Millam, J. M.; Iyengar, S. S.; Tomasi, J.; Barone, V.; Mennucci, B.; Cossi, M.; Scalmani, G.; Rega, N.; Petersson, G. A.; Nakatsuji, H.; Hada, M.; Ehara, M.; Toyota, K.; Fukuda, R.; Hasegawa, J.; Ishida, M.; Nakajima, T.; Honda, Y.; Kitao, O.; Nakai, H.; Klene, M.; Li, X.; Knox, J. E.; Hratchian, H. P.; Cross, J. B.; Adamo, C.; Jaramillo, J.; Gomperts, R.; Stratmann, R. E.; Yazyev, O.; Austin, A. J.; Cammi, R.; Pomelli, C.; Ochterski, J. W.; Ayala, P. Y.; Morokuma, K.; Voth, G. A.; Salvador, P.; Dannenberg, J. J.; Zakrzewski, V. G.; Dapprich, S.; Daniels, A. D.; Strain, M. C.; Farkas, O.; Malick, D. K.; Rabuck, A. D.; Raghavachari, K.; Foresman, J. B.; Ortiz, J. V.; Cui, Q.; Baboul, A. G.; Clifford, S.; Cioslowski, J.; Stefanov, B. B.; Liu, G.; Liashenko, A.; Piskorz, P.; Komaromi, I.; Martin, R. L.; Fox, D. J.; Keith, T.; M. A. Al-Laham, Peng, C. Y.; Nanayakkara, A.; Challacombe, M.; Gill, P. M. W.; Johnson, B.; Chen, W.; Wong, M. W.; Gonzalez, C.; Pople, J. A., Gaussian, Inc.: Pittsburgh, PA, 2003.

(31) Chaumont, A.; Wipff, G. J. Phys. Chem. B 2010, 114, 13773.

(32) Vayssière, P.; Chaumont, A.; Wipff, G. Phys. Chem. Chem. Phys. 2005, 7, 124.

(33) Schurhammer, R.; Vayssière, P.; Wipff, G. J. Phys. Chem. A 2003, $107,11128$.

(34) Vayssière, P.; Wipff, G. Phys. Chem. Chem. Phys. 2003, 5, 127.

(35) Vayssière, P.; Wipff, G. Phys. Chem. Chem. Phys. 2003, 5, 2842.

(36) Buhl, M.; Wipff, G. J. Am. Chem. Soc. 2002, 124, 4473.

(37) Visser, A. E.; Swatloski, R. P.; Reichert, W. M.; Griffin, S. T.; Rogers, R. D. Ind. Eng. Chem. Res. 2000, 39, 3596.

(38) Dietz, M. L.; Dzielawa, J. A. Chem. Commun. 2001, 2124.

(39) Steed, J. W. Coord. Chem. Rev. 2001, 215, 171.

(40) Bradshaw, J. S.; Izatt, R. M. Acc. Chem. Res. 1997, 30, 38.

(41) Schlitter, J. Chem. Phys. Lett. 1993, 215, 617.

(42) Schäfer, H.; Mark, H. E.; van Gunsteren, W. F. J. Chem. Phys. 2000, 113, 780 .

(43) Alemán, C.; Curcó, D.; Casanovas, J. Phys. Rev. E 2005, 72, 026704.
(44) Alemán, C.; Curcó, D.; Casanovas, J. Chem. Phys. Lett. 2004, $386,408$. 